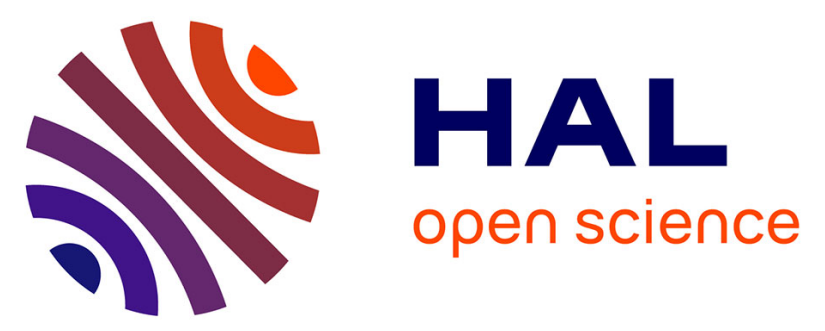

\title{
Development of 55 novel polymorphic microsatellite loci for the critically endangered L. (Actinopterygii: Perciformes: Percidae) and cross-species amplification in five other percids
}

Vincent Dubut, Rémi Grenier, Emese Meglécz, Rémi Chappaz, Caroline Costedoat, Delphine Danancher, Stéphane Descloux, Thibaut Malausa, Jean-François Martin, Nicolas Pech, et al.

\section{- To cite this version:}

Vincent Dubut, Rémi Grenier, Emese Meglécz, Rémi Chappaz, Caroline Costedoat, et al.. Development of 55 novel polymorphic microsatellite loci for the critically endangered L. (Actinopterygii: Perciformes: Percidae) and cross-species amplification in five other percids. European Journal of Wildlife Research, 2010, 56 (6), pp.931-938. 10.1007/s10344-010-0421-x . hal-00609749

\section{HAL Id: hal-00609749 https://hal.science/hal-00609749}

Submitted on 20 Jul 2011

HAL is a multi-disciplinary open access archive for the deposit and dissemination of scientific research documents, whether they are published or not. The documents may come from teaching and research institutions in France or abroad, or from public or private research centers.
L'archive ouverte pluridisciplinaire HAL, est destinée au dépôt et à la diffusion de documents scientifiques de niveau recherche, publiés ou non, émanant des établissements d'enseignement et de recherche français ou étrangers, des laboratoires publics ou privés. 


\title{
Development of 55 novel polymorphic microsatellite loci for the critically endangered Zingel asper L. (Actinopterygii: Perciformes: Percidae) and cross-species amplification in five other percids
}

\author{
Vincent Dubut • Rémi Grenier • Emese Meglécz • Rémi Chappaz • \\ Caroline Costedoat • Delphine Danancher • Stéphane Descloux • Thibaut Malausa • \\ Jean-François Martin • Nicolas Pech • André Gilles
}

Received: 19 November 2009 /Revised: 5 July 2010 / Accepted: 6 July 2010 / Published online: 20 July 2010

(C) Springer-Verlag 2010

\begin{abstract}
By combining biotin-enrichment protocol and next generation pyrosequencing, through 454 GS-FLX Titanium technology, 55 polymorphic microsatellites loci with perfect motif were isolated from the Rhone streber (Zingel asper), a critically endangered European fish species. Eight multiplex PCR kits were optimised in order to genotype a total of 58 polymorphic loci, including three previously published loci. The level of genetic diversity was assessed for 68 Z. asper, 30 Sander lucioperca, 33 Perca fluviatilis and four Gymnocephalus schraetzer individuals. Amplification success was also assessed on Romanichthys valsanicola and Zingel streber using single individuals. These markers will be useful to investigate the population structure of the highly fragmented Rhone streber. They represent a powerful tool for conservation issues and evolutionary approaches of this endemic species.
\end{abstract}

Communicated by C. Gortázar

V. Dubut $(\bowtie) \cdot$ R. Grenier $\cdot$ E. Meglécz $\cdot$ R. Chappaz $\cdot$

C. Costedoat $\cdot$ N. Pech $\cdot$ A. Gilles

Aix-Marseille Université, CNRS, IRD, UMR 6116-IMEP,

Equipe Evolution Génome Environnement,

Centre Saint-Charles, Case 36, 3 place Victor Hugo,

13331 Marseille Cedex 3, France

e-mail: vincent.dubut@univ-provence.fr

D. Danancher

Conservatoire Rhône-Alpes des Espaces Naturels,

Maison Forte, 2 rue des Vallières,

69390 Vourles, France

\section{Danancher}

Université Lyon I, CNRS, UMR 5023-LEHF,

Equipe Biodiversité des Ecosystèmes Lotiques,

43 boulevard du 11 novembre 1918 ,

69622 Villeurbanne Cedex, France
Moreover, part of our markers demonstrated applicability to other percid species, allowing for potential applications to fisheries and aquaculture management.

Keywords Percidae $\cdot$ STRs $\cdot$ Enriched library Pyrosequencing · QDD · Conservation genetics

\section{Introduction}

The Rhone streber, Zingel asper (Linnaeus, 1758) [Actinopterygii: Perciformes: Percidae], is defined as a critically endangered species by the International Union for Conservation of Nature. Since the beginning of the twentieth century, Z. asper declined by about $80 \%$ of its initial distribution range as a result of combined anthropogenic

\section{S. Descloux}

Electricité de France, Service Environnement et Société,

Centre d'Ingénierie Hydraulique,

Savoie-Technolac,

73373 Le Bourget-du-Lac, France

T. Malausa

INRA, CNRS, Université de Nice-Sophia Antipolis,

UMR 1301-IBSV, Equipe Biologie des Populations en Interactions,

400 route des Chappes, BP 167, 06903 Sophia-Antipolis Cedex,

France

J.-F. Martin

UMR CBGP (INRA/IRD/CIRAD/Montpellier SupAgro),

Montpellier SupAgro,

Campus international de Baillarguet, CS 30016, 34988

Montferrier-sur-Lez Cedex, France 
factors: habitat fragmentation, hydraulic disturbance and pollution. This species is now restricted to a few disconnected populations while it was formerly present in the whole Rhone River drainage (Mari et al. 2002). Whether this geographic shrinkage and habitat fragmentations caused a loss of genetic diversity and genetic drift within isolated populations remains unclear (but see Laroche and Durand 2004). Since conservation strategies rely on the understanding of population structure and dynamics, analysing the genetic structure of the Rhone streber with fine-scale molecular markers such as microsatellites would provide useful information. The development of microsatellite markers would allow for delineating the evolutionary history of the species and evaluating the genetic diversity in both evolutionary and conservation frameworks (as initiated in Danancher et al. 2008).

In this study, we detail the development of 58 polymorphic microsatellites for the Rhone streber (including 55 novel markers with perfect motif) using a combination of biotin-enrichment and 454 pyrosequencing technology. We also test for the applicability of these markers on five other percid species (Sander lucioperca, Perca fluviatilis, Gymnocephalus schraetzer, Romanichthys valsanicola and Zingel streber).

\section{Material and methods}

Caudal fin samples were collected from 68 individuals of $Z$. asper in Durance River (southeastern France; $n=36$ ) and Beaume River (southeastern France; $n=32$ ). Additionally, individuals from five other Percidae species were collected: $30 \mathrm{~S}$. lucioperca individuals (Rhone delta, southeastern France), 33 P. fluviatilis (Durance catchment), one $R$. valsanicola (Valsan River, Romania), one Z. streber and four G. schraetzer (Danube drainage).

Microsatellites were isolated following a biotinenrichment protocol adapted from Kijas et al. (1994) at Genoscreen (Lille, France). DNA was extracted from samples, digested with $R s a$ I (Fermentas) and then ligated to standard oligonucleotide adapters as described elsewhere (Dubut et al. 2009). Three biotin-labelled oligonucleotides- $-(\mathrm{TG})_{10},(\mathrm{TC})_{13}$ and $(\mathrm{AAC})_{7} \mathrm{~A}$-were independently hybridised to the ligated DNA at $56^{\circ} \mathrm{C}$ for $20 \mathrm{~min}$ after initial denaturation of the ligation. The enrichment step was completed using Dynabeads (Invitrogen) and standard procedure. The resulting enriched DNA was amplified using standard adapters, with 25 cycles $\left(20 \mathrm{~s}\right.$ at $95^{\circ} \mathrm{C}, 20 \mathrm{~s}$ at $60^{\circ} \mathrm{C}$ and $1 \mathrm{~min} 30 \mathrm{~s}$ at $72^{\circ} \mathrm{C}$ ) and a final elongation step of $30 \mathrm{~min}$ at $72^{\circ} \mathrm{C}$. The amplicons were immediately purified using QIAquick PCR Purification Kit (QIAGEN). Each purified enriched library was used in the GS-FLX 454 Titanium (Roche
Applied Science) library preparation following the manufacturer's protocols at Genoscreen (Lille, France).

Emulsion PCR was carried out at a ratio of 1 copy per bead and subsequently disrupted using isopropanol. Beads containing amplified DNA fragments were enriched and recovered for sequencing to finally provide 50,000 to 70,000 enriched beads for each library. The recovered ssDNA beads were packed onto $1 / 16$ region of a $70 \times 75-\mathrm{mm}$ Titanium PicoTiter plate and sequenced with 200 cycles. Sample preparation and analytical processing such as base calling were performed at Genoscreen (Lille, France) using the manufacturer's protocol for Titanium series.

The selection of sequences for primer design was done with the programme QDD (Meglécz et al. 2010). Enrichment adaptors were removed from sequences. Sequences longer than $80 \mathrm{bp}$ and containing perfect microsatellite motif of at least five repetitions for any microsatellite motif of 2-6 bp were selected for further analyses. Sequence similarities were computed through an 'all against all' BLAST (Altschul et al. 1997; $e$ value 1E-40) in which microsatellite motifs were soft masked. Sequences for which pairwise similarity along the flanking regions was over $95 \%$ were grouped into contigs, and a $2 / 3$ majority rule consensus sequence was created from each contig. Sequences which had significant BLAST hit to other sequences with an overall similarity among the flanking region bellow $95 \%$ were discarded to avoid potential intragenomic multicopy sequences. All unique sequences (with no BLAST hit to any other ' 454 ' reads) and consensus sequences were checked for the presence of short repetitions in the flanking regions. PCR primers were designed using QDD with the following criteria: (a) target microsatellite displayed at least five repetitions, (b) length of PCR product was between 100 and $300 \mathrm{bp}$, (c) flanking regions contained at most one mononucleotide stretch of four bases and two repetitions of any di-hexa base pair motifs and (d) annealing temperatures of primers pairs were optimised for falling between $57^{\circ} \mathrm{C}$ and $63^{\circ} \mathrm{C}$. Among the 241 sequences for which primer pairs could be designed, we selected 181 sequences by discarding 60 sequences with only five or six repeats.

Amplifications were performed for each of the 181 primers pairs using reagents and protocols described previously (Dubut et al. 2009). The 105 novel primer pairs displaying specific PCR products on agarose gel electrophoresis were retained for further analyses. At this stage, primers pairs of three previously described microsatellite loci (Svi18: Borer et al. 1999; SviL8 and SviL9: Wirth et al. 1999) were included into the protocol. All the 108 loci were then amplified separately using forward primers labelled with fluorescent dyes 6-FAM (Eurogentec), PET, NED or VIC (Applied Biosystems). Visualisation of the amplicons was conducted on an ABI 3130XL Genetic Analyzer 
Table 1 Microsatellite loci and multiplex PCR conditions

\begin{tabular}{|c|c|c|c|c|c|}
\hline \multirow{2}{*}{$\begin{array}{l}\text { Locus } \\
\text { (GenBank ID) }\end{array}$} & \multirow{2}{*}{$\begin{array}{l}\text { Fluorescent } \\
\text { dye }\end{array}$} & \multirow[t]{2}{*}{ Primer sequence $\left(5^{\prime}-3^{\prime}\right)$} & \multirow[t]{2}{*}{ Core motif } & \multicolumn{2}{|c|}{ Multiplex PCR } \\
\hline & & & & Kit & $\begin{array}{l}\text { Primers } \\
\text { concentration } \\
(\mathrm{nM})\end{array}$ \\
\hline Svi18 (G36964) & NED & $\begin{array}{l}\text { F: GATCTGTAAACTCCAGCGTG } \\
\text { R: CTTAAGCTGCTCAGCATCCAGG }\end{array}$ & $(\mathrm{AC})_{11}$ & 1 & 400 \\
\hline SviL8 (AF144741) & VIC & $\begin{array}{l}\text { F: GCTTATACGTCGTTCTTATG } \\
\text { R: ATGGAGAAGCAAGTTGAG }\end{array}$ & $(\mathrm{TG})_{22}$ & 1 & 150 \\
\hline SviL9 (AF144742) & 6-FAM & $\begin{array}{l}\text { F: TACTGTTCACTTATCTATCC } \\
\text { R: TGTATGTGTGTGTGTTCATGT }\end{array}$ & $(\mathrm{CA})_{18} \mathrm{AA}(\mathrm{CA})_{3} \mathrm{~A}(\mathrm{AC})_{4}$ & 1 & 400 \\
\hline Za022 (HM622293) & VIC & $\begin{array}{l}\text { F: AGTCGATGCTTGATGCTGTG } \\
\text { R: GAGAAGGAAGGAGAAAGAGATGA }\end{array}$ & $(\mathrm{CT})_{10}$ & 1 & 50 \\
\hline Za097 (HM622309) & PET & $\begin{array}{l}\text { F: TATTGAACGAAGAGCTGCGG } \\
\text { R: TCGGGTTATTTAACCGGCTC }\end{array}$ & $(\mathrm{GA})_{8}$ & 1 & 100 \\
\hline Za118 (HM622315) & NED & $\begin{array}{l}\text { F: TTGACACATATCATGAACCACTACA } \\
\text { R: GACCGCTGGTTCTTCTTCAG }\end{array}$ & $(\mathrm{GTT})_{9}$ & 1 & 50 \\
\hline Za190 (HM622332) & PET & $\begin{array}{l}\text { F: CATTCCATTTTGTTGGCTCA } \\
\text { R: GCTTTGGAAAGAAACGATGG }\end{array}$ & $(\mathrm{TGA})_{10}$ & 1 & 100 \\
\hline Za215 (HM622339) & 6-FAM & $\begin{array}{l}\text { F: ACGCATCTGTCCACCACTG } \\
\text { R: CCTCCCCACTGGATAGGAAT }\end{array}$ & $(\mathrm{AC})_{7}$ & 1 & 100 \\
\hline Za143 (HM622318) & VIC & $\begin{array}{l}\text { F: TAAGTGTTAAAATTGTAGCCCATTTC } \\
\text { R: GGACATTTCTAAGTGACCCCA }\end{array}$ & $(\mathrm{CA})_{12}$ & 2 & 250 \\
\hline Za176 (HM622327) & PET & $\begin{array}{l}\text { F: AGTGGTTACTTGTGGAGCGG } \\
\text { R: GATTTCCGATTACCTGGAACA }\end{array}$ & $(\mathrm{CTT})_{8}$ & 2 & 250 \\
\hline Za179 (HM622329) & NED & $\begin{array}{l}\text { F: ATTTCCCATTGCGGGATTA } \\
\text { R: GGATTCTTGCATGCTTTGGT }\end{array}$ & $(\mathrm{TCT})_{9}$ & 2 & 50 \\
\hline Za186 (HM622331) & 6-FAM & $\begin{array}{l}\text { F: CAGCTTGCAGTGAAAGACCA } \\
\text { R: TGGAGAGTGGATGGATTTACAG }\end{array}$ & $(\mathrm{AC})_{10}$ & 2 & 250 \\
\hline Za207 (HM622337) & VIC & $\begin{array}{l}\text { F: GGATTCCAGAAGCAAAAGAGG } \\
\text { R: TGGGACAAGGCTTTAACCAC }\end{array}$ & $(\mathrm{GT})_{13}$ & 2 & 100 \\
\hline Za209 (HM622338) & NED & $\begin{array}{l}\text { F: CATCACTGTCCAGCAAAAGC } \\
\text { R: ATGTGAATTTCCCTTGTGGG }\end{array}$ & $(\mathrm{GAA})_{8}$ & 2 & 40 \\
\hline Za222 (HM622341) & NED & $\begin{array}{l}\text { F: TCTCTGTCACAGGAGGTCCA } \\
\text { R: ATTACCGGTTTGAAGGGCTG }\end{array}$ & $(\mathrm{AAGGAG})_{5}$ & 2 & 75 \\
\hline Za040 (HM622299) & PET & $\begin{array}{l}\text { F: TTTGTGATATTCCACTCTGAAAGC } \\
\text { R: TGGCATAATTCAGCCATCAA }\end{array}$ & $(\mathrm{CT})_{11}$ & 3 & 100 \\
\hline Za107 (HM622313) & NED & $\begin{array}{l}\text { F: CCTGTACACGTTATTCCCCA } \\
\text { R: CTTCAGCACTGTATTGAGGGG }\end{array}$ & $(\mathrm{GA})_{17}$ & 3 & 100 \\
\hline Za113 (HM622314) & PET & $\begin{array}{l}\text { F: ACCACGCACAATCACTCGTA } \\
\text { R: CCTGGCTTTACCCAGAAACA }\end{array}$ & $(\mathrm{CA})_{11}$ & 3 & 100 \\
\hline Za144 (HM622319) & NED & $\begin{array}{l}\text { F: GCCCACAATAGCACCGTAAT } \\
\text { R: TTTGTGAATGTGAGTGAGAGTCAG }\end{array}$ & $(\mathrm{AC})_{8}$ & 3 & 50 \\
\hline Za161 (HM622322) & VIC & $\begin{array}{l}\text { F: AACCAATTTCAGTCCATTCTTCA } \\
\text { R: CAAAGAAACAAAACAACTGTTCC }\end{array}$ & $(\mathrm{TG})_{9}$ & 3 & 250 \\
\hline Za164 (HM622323) & 6-FAM & $\begin{array}{l}\text { F: TTCATGCAAGTTTGCCGTT } \\
\text { R: TGGTTGCCAATCACTGATCTA }\end{array}$ & $(\mathrm{GAA})_{5}$ & 3 & 150 \\
\hline Za165 (HM622324) & VIC & $\begin{array}{l}\text { F: GTTGTCTCAAATCACGCACG } \\
\text { R: TTGTCTGGCAACAATAGGTGA }\end{array}$ & $(\mathrm{TCT})_{8}$ & 3 & 50 \\
\hline Za200 (HM622335) & 6-FAM & $\begin{array}{l}\text { F: GTTAGCGTGCCAAGTTGATG } \\
\text { R: GTAGGTGGCGGTATGCATTT }\end{array}$ & $(\mathrm{TTC})_{11}$ & 3 & 100 \\
\hline $\mathrm{Za030}$ (HM622296) & NED & $\begin{array}{l}\text { F: TTTGTAGGGTGTTTAAAGTTTGC } \\
\text { R: ATTTTCTGCAGTGGTCCCAG }\end{array}$ & $(\mathrm{GA})_{9}$ & 4 & 50 \\
\hline Za032 (HM622297) & VIC & $\begin{array}{l}\text { F: GAGATGACATGCCTTGCCTC } \\
\text { R: CACAAACTAAAGACCAACAAACCA }\end{array}$ & $(\mathrm{TG})_{10}$ & 4 & 150 \\
\hline
\end{tabular}


Table 1 (continued)

\begin{tabular}{|c|c|c|c|c|c|}
\hline \multirow{2}{*}{$\begin{array}{l}\text { Locus } \\
\text { (GenBank ID) }\end{array}$} & \multirow{2}{*}{$\begin{array}{l}\text { Fluorescent } \\
\text { dye }\end{array}$} & \multirow[t]{2}{*}{ Primer sequence $\left(5^{\prime}-3^{\prime}\right)$} & \multirow[t]{2}{*}{ Core motif } & \multicolumn{2}{|c|}{ Multiplex PCR } \\
\hline & & & & Kit & $\begin{array}{l}\text { Primers } \\
\text { concentration } \\
(\mathrm{nM})\end{array}$ \\
\hline Za078 (HM622303) & PET & $\begin{array}{l}\text { F: ATTATGACGCGTATCCCACG } \\
\text { R: AAAAGGAAGATGTGCACAGTGA }\end{array}$ & $(\mathrm{GA})_{9}$ & 4 & 50 \\
\hline Za091 (HM622306) & NED & $\begin{array}{l}\text { F: TTCTGATTTAGCCCTGCCTG } \\
\text { R: GTAGGCAGAAATCCTGCACC }\end{array}$ & $(\mathrm{TG})_{5}$ & 4 & 50 \\
\hline Za094 (HM622307) & 6-FAM & $\begin{array}{l}\text { F: CAGACACAAACAACACAACTGG } \\
\text { R: CAACACTGTATGGTCATAAATAGCAA }\end{array}$ & $(\mathrm{GATA})_{11}$ & 4 & 250 \\
\hline Za096 (HM622308) & 6-FAM & $\begin{array}{l}\text { F: AAGATTTTGGTGTTGGCGAG } \\
\text { R: TACTTGTCCTTTCCGGGTTG }\end{array}$ & $(\mathrm{GA})_{17}$ & 4 & 300 \\
\hline Za106 (HM622312) & PET & $\begin{array}{l}\text { F: ATTACGTTTGGGGAAGAGGC } \\
\text { R: AGTTGCTTTCATTTCCCTGC }\end{array}$ & $(\mathrm{AG})_{8}$ & 4 & 75 \\
\hline Za121 (HM622316) & PET & $\begin{array}{l}\text { F: CAAAGTCATGAACGAGCTGC } \\
\text { R: AGCCAGGACCACTCTGTGAG }\end{array}$ & $(\mathrm{CT})_{9}$ & 4 & 150 \\
\hline Za241 (HM622344) & NED & $\begin{array}{l}\text { F: ATTTCAATGTGGTGCAAATTAGC } \\
\text { R: TGCGCACATACATGCATAG }\end{array}$ & $(\mathrm{GT})_{10}$ & 4 & 100 \\
\hline Za006 (HM622290) & 6-FAM & $\begin{array}{l}\text { F: TGGTGGCACAAATCTCCATA } \\
\text { R: CCTGTAATGATGGCTTGAGGA }\end{array}$ & $(\mathrm{AG})_{7}$ & 5 & 100 \\
\hline Za038 (HM622298) & NED & $\begin{array}{l}\text { F: TGAATCGCTGCTCTTTCTCA } \\
\text { R: TATGCAATTACATCGGAGCG }\end{array}$ & $(\mathrm{AC})_{11}$ & 5 & 100 \\
\hline Za069 (HM622300) & VIC & $\begin{array}{l}\text { F: AATTCTAAGACAATGTTTCTTTCATCT } \\
\text { R: CACATCAATGGTTGCTTCTTG }\end{array}$ & $(\mathrm{TG})_{8}$ & 5 & 75 \\
\hline $\mathrm{Za072}$ (HM622301) & VIC & $\begin{array}{l}\text { F: TTTAAACAAATACACTCAGAGGGA } \\
\text { R: TCCCCTATCTCTTCTTTATTTCACC }\end{array}$ & $(\mathrm{GA})_{8}$ & 5 & 150 \\
\hline Za102 (HM622311) & NED & $\begin{array}{l}\text { F: CACCAGCGACAAACAAACAC } \\
\text { R: GCAAACTGTTCCAGCTCCTC }\end{array}$ & $(\mathrm{TC})_{5}$ & 5 & 40 \\
\hline Za138 (HM622317) & PET & $\begin{array}{l}\text { F: TTCTTTATACAAGAGGAATAGTTGCAG } \\
\text { R: TTTTTGTGATTGTGCTATTTTAAAGG }\end{array}$ & $(\mathrm{AC})_{8}$ & 5 & 200 \\
\hline Za169 (HM622325) & PET & $\begin{array}{l}\text { F: CCCACTGTGTCATCGTCATC } \\
\text { R: GAAGAAAATCATAAATGACAACTGC }\end{array}$ & $(\mathrm{CTT})_{6}$ & 5 & 50 \\
\hline Za170 (HM622326) & 6-FAM & $\begin{array}{l}\text { F: GGAACACGTCTGTTTTGCTG } \\
\text { R: CTTGCAACAGCATCAGTGGT }\end{array}$ & $(\mathrm{CTT})_{10}$ & 5 & 50 \\
\hline Za194 (HM622333) & VIC & $\begin{array}{l}\text { F: AGTGCTGCTTCTGTGGAGGT } \\
\text { R: CAGCCTGTGCTGGGAATAAT }\end{array}$ & $(\mathrm{TGG})_{5}$ & 5 & 50 \\
\hline Za011 (HM622291) & VIC & $\begin{array}{l}\text { F: CCATCAAATCACATAGCTGGA } \\
\text { R: TCTTTGCTTGCGTCACCTAA }\end{array}$ & $(\mathrm{AG})_{8}$ & 6 & 50 \\
\hline Za019 (HM622292) & PET & $\begin{array}{l}\text { F: CTTAGCCAGACGTTGCCTGT } \\
\text { R: AATTTGTTTGCGTCTGGTCA }\end{array}$ & $(\mathrm{AC})_{7}$ & 6 & 100 \\
\hline Za086 (HM622304) & 6-FAM & $\begin{array}{l}\text { F: CAATGCCACTGGGCTAAATC } \\
\text { R: GCTGTCTGGATAATGTTTCAACT }\end{array}$ & $(\mathrm{AG})_{10}$ & 6 & 600 \\
\hline Za154 (HM622320) & NED & $\begin{array}{l}\text { F: GCCATGTTCTTTATTCACTTGC } \\
\text { R: CCAACGAGCCTTCTCTGAAC }\end{array}$ & $(\mathrm{GTT})_{7}$ & 6 & 50 \\
\hline Za199 (HM622334) & PET & $\begin{array}{l}\text { F: CCTTCCCCTCAAAAGCATGT } \\
\text { R: AGGAAATGGAAAGGGAATGC }\end{array}$ & $(\mathrm{TCT})_{13}$ & 6 & 100 \\
\hline Za221 (HM622340) & 6-FAM & $\begin{array}{l}\text { F: CATTTCACCGCATTCAGTTG } \\
\text { R: AAAACCACGAAATACAGCGG }\end{array}$ & $(\mathrm{TCT})_{8}$ & 6 & 100 \\
\hline Za024 (HM622294) & VIC & $\begin{array}{l}\text { F: TGAACCTCCCTATCCCCTCT } \\
\text { R: TCTTTTCCACAGCAGGAAGC }\end{array}$ & $(\mathrm{AC})_{7}$ & 7 & 40 \\
\hline Za087 (HM622305) & 6-FAM & $\begin{array}{l}\text { F: TCCCTGTCGTCAAAGACCTC } \\
\text { R: GACGTCAATGGACTGTTCCC }\end{array}$ & $(\mathrm{AC})_{9}$ & 7 & 150 \\
\hline Za101 (HM622310) & PET & $\begin{array}{l}\text { F: ATCAAGGTCCAGAGGCAAGA } \\
\text { R: AACAGATGGTCCTCTGGCTG }\end{array}$ & $(\mathrm{AG})_{10}$ & 7 & 200 \\
\hline
\end{tabular}


Table 1 (continued)

\begin{tabular}{|c|c|c|c|c|c|}
\hline \multirow{2}{*}{$\begin{array}{l}\text { Locus } \\
\text { (GenBank ID) }\end{array}$} & \multirow{2}{*}{$\begin{array}{l}\text { Fluorescent } \\
\text { dye }\end{array}$} & \multirow[t]{2}{*}{ Primer sequence $\left(5^{\prime}-3^{\prime}\right)$} & \multirow[t]{2}{*}{ Core motif } & \multicolumn{2}{|c|}{ Multiplex PCR } \\
\hline & & & & Kit & $\begin{array}{l}\text { Primers } \\
\text { concentration } \\
(\mathrm{nM})\end{array}$ \\
\hline Za157 (HM622321) & NED & $\begin{array}{l}\text { F: TCAGACTTGGAGAAGGAGGG } \\
\text { R: TCAGTCTCTTTCATCGCTGC }\end{array}$ & $(\mathrm{GAA})_{7}$ & 7 & 40 \\
\hline Za181 (HM622330) & 6-FAM & $\begin{array}{l}\text { F: TGGACTTTTCACCACAGCAG } \\
\text { R: GGAGGAGGTGGTCAAAGAGA }\end{array}$ & $(\mathrm{TCA})_{6}$ & 7 & 100 \\
\hline Za203 (HM622336) & PET & $\begin{array}{l}\text { F: AGCATCCGGTTCTTTACCTG } \\
\text { R: TGGCTGGTATTGAGTGGTAGG }\end{array}$ & $(\mathrm{AC})_{8}$ & 7 & 100 \\
\hline Za029 (HM622295) & NED & $\begin{array}{l}\text { F: TCCTGCTCTAACAGGTGCAA } \\
\text { R: CAGACGCACTGATCACCAAT }\end{array}$ & $(\mathrm{AG})_{7}$ & 8 & 75 \\
\hline $\mathrm{Za077} \mathrm{(HM622302)}$ & 6-FAM & $\begin{array}{l}\text { F: TCTAGCCACCAAATGACCA } \\
\text { R: TTTCACCATCTACGTTTTGTTCTT }\end{array}$ & $(\mathrm{GT})_{8}$ & 8 & 500 \\
\hline Za178 (HM622328) & PET & $\begin{array}{l}\text { F: AGGAGGCAGTAATGCAATGT } \\
\text { R: GCACTGATGTAAGTGCAATAGGC }\end{array}$ & $(\mathrm{AAG})_{7}$ & 8 & 100 \\
\hline Za237 (HM622342) & 6-FAM & $\begin{array}{l}\text { F: ATCTCAAGTCATGGGGCATC } \\
\text { R: GGTCCTCTGGTGCAGCTATAA }\end{array}$ & $(\mathrm{CA})_{10}$ & 8 & 150 \\
\hline Za239 (HM622343) & NED & $\begin{array}{l}\text { F: CGAAGTATATTCAGATAGACCCT } \\
\text { R: CTGTCTTTGTCATAAACTTAACTTACTC }\end{array}$ & $(\mathrm{GA})_{10}$ & 8 & 100 \\
\hline
\end{tabular}

(Applied Biosystems). Alleles sizes were scored against the GeneScan-500 LIZ ${ }^{\circledR}$ Size Standard (Applied Biosystems), and genotypes were obtained using GeneMapper ${ }^{\circledR} \quad 3.7$ (Applied Biosystems). A total of 58 primers pairs associated with an unambiguous genotype profile in $Z$. asper were selected and combined into eight PCR multiplex kits (Table 1). The multiplex kits were further tested on six percid species (including Z. asper).

GENEPOP 4.0 (Rousset 2008) was used to (a) test HardyWeinberg (HW) equilibrium, (b) estimate the heterozygosity for all loci and populations and (c) test for linkage disequilibrium (LD) among loci within populations.

\section{Results}

Concerning $Z$. asper, the number of alleles per locus ranged from 2 to 25, and expected heterozygosity values ranged from 0.03 to 0.95 (Table 2). After FDR correction (modified as proposed by Benjamini and Hochberg 1995), all but two loci (SviL8 and $\mathrm{Za038}$ in the Durance sample, with significant excess of homozygotes) displayed HW equilibrium (Table 2), and significant LD among loci was detected between two loci (Za207 and Za239) in the Beaume sample only.

As for $S$. lucioperca and P. fluviatilis, the amplification revealed successful for 47 and 35 loci, respectively (Table 2). Eighteen loci were polymorphic in S. lucioperca (although polymorphic, SviL9 was disregarded as homozygotes with null allele were detected), and 14 were polymorphic in $P$. fluviatilis. All polymorphic loci did not depart from HW equilibrium (except $\mathrm{Za072}$ in S. lucioperca), and no LD was detected, except for one pair of loci (Za121 vs. Za144 in S. lucioperca). In S. lucioperca, the number of alleles per polymorphic locus ranged from 2 to 9 , and expected heterozygosity values ranged from 0.32 to 0.85 . In $P$. fluviatilis, the number of alleles per polymorphic locus ranged from 2 to 14, and expected heterozygosity values ranged from 0.06 to 0.88 (Table 2). Additionally, cross-species amplifications were successful in 53.4\% cases for $G$. schraetzer, in $69.0 \%$ cases for $R$. valsanicola and in $70.7 \%$ cases for Z. streber (Table 2).

\section{Discussion}

The combination of biotin-enrichment protocol and next generation pyrosequencing, through 454 GS-FLX Titanium technology allowed the fast development of polymorphic markers for the threatened Z. asper. The 55 new markers developed here (along with three previously published markers) constitute a useful tool for achieving detailed information on the genetic structure of this species and investigating its evolutionary history. Furthermore, it will assist in the development of comprehensive long-term management and protection plans. In this context, normalisation of the PCR conditions and multiplexing make faster and cost effective the genotyping of the 58 loci. Moreover, all the novel 55 markers display perfect microsatellite motif. This makes them easily usable not only for 


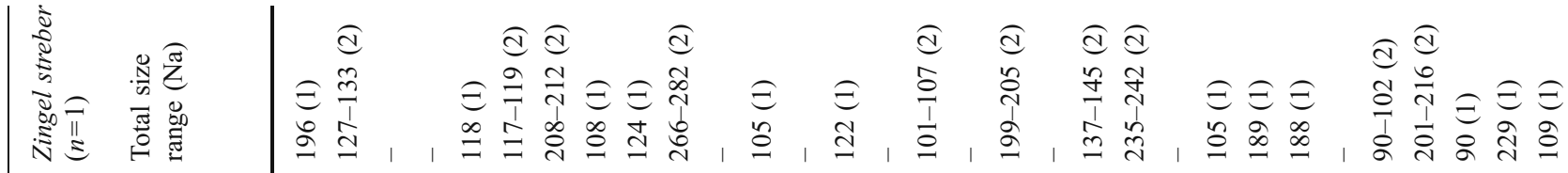

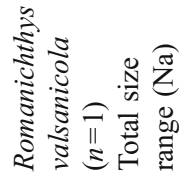

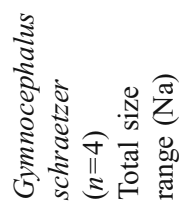

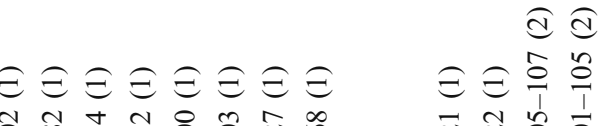

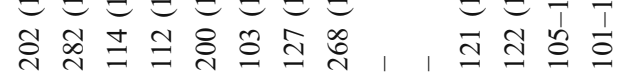

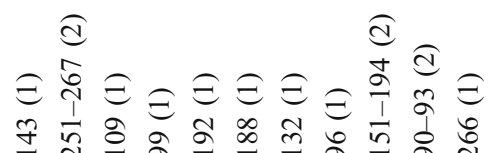

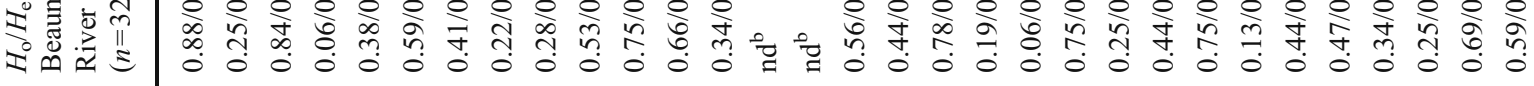

于

c)

(a)

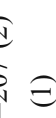

(2)

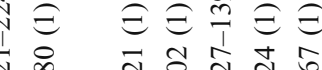

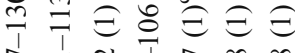

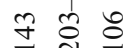

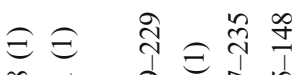

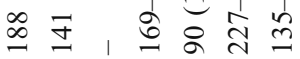

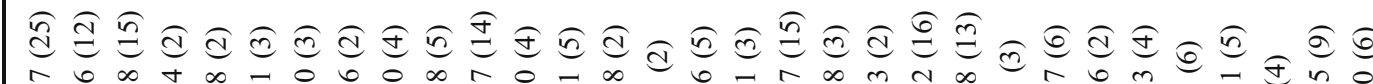

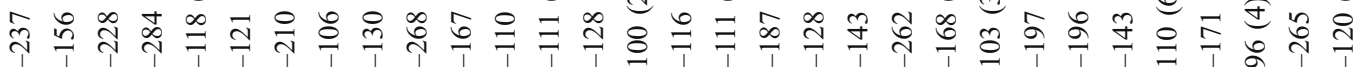

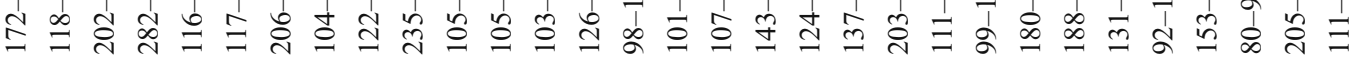

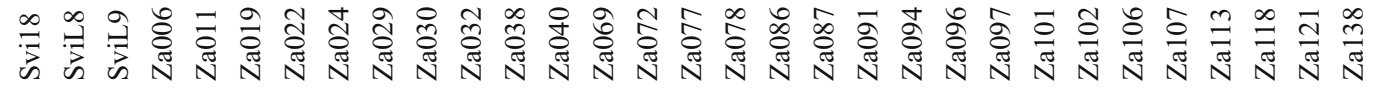




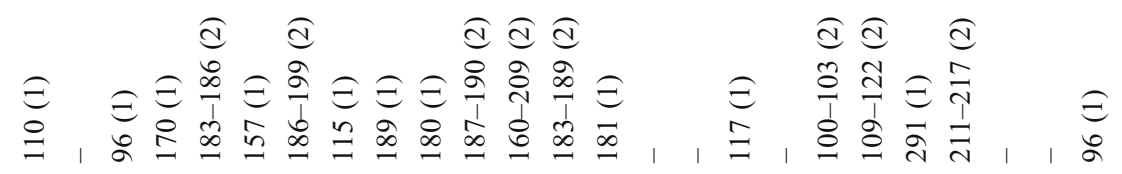

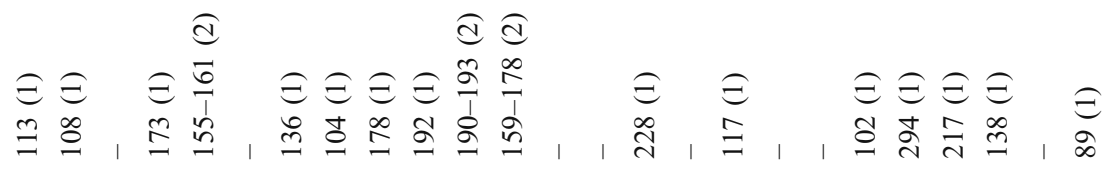

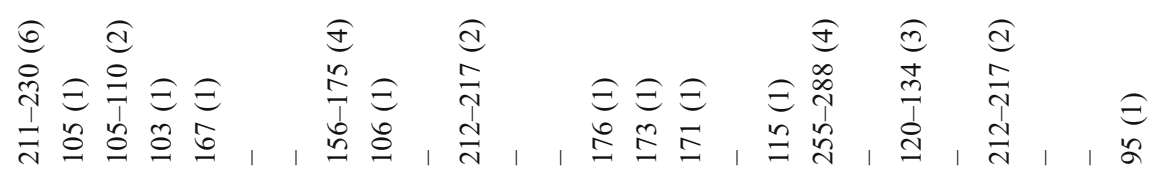

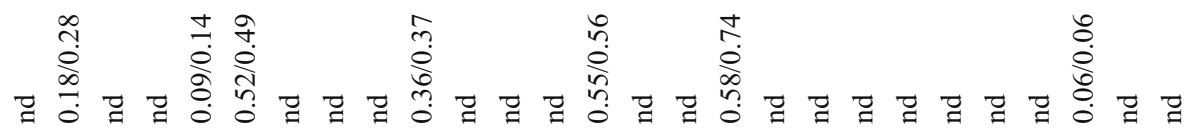

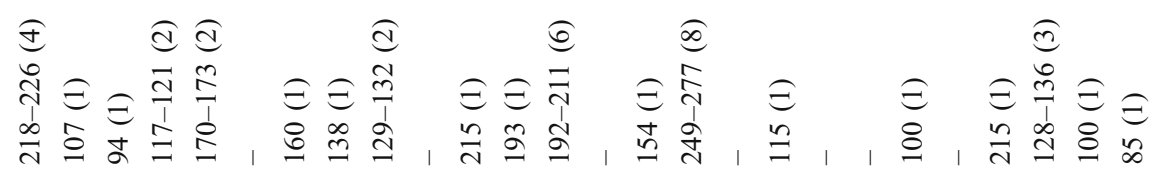

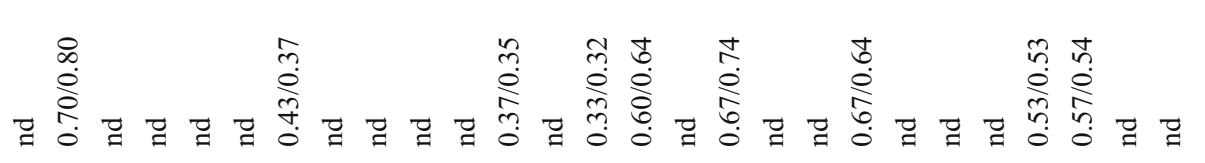

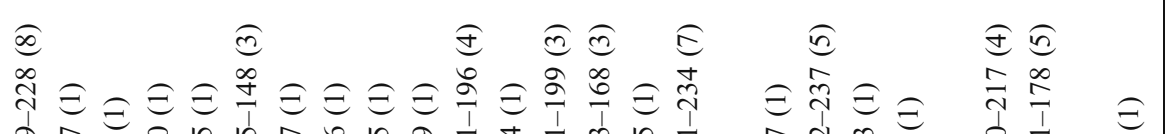

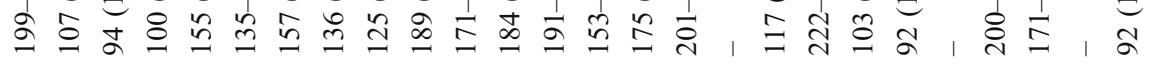

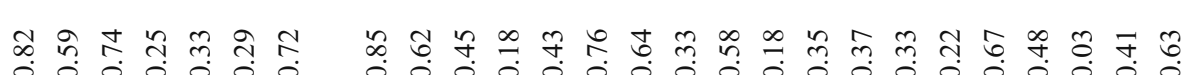

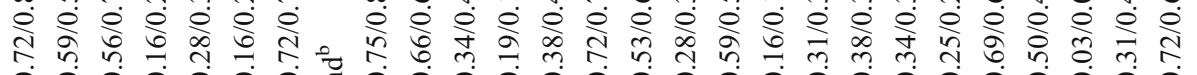

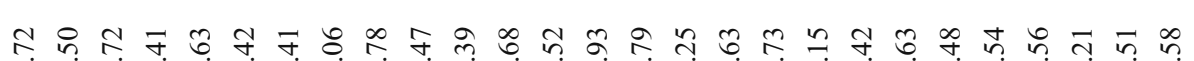

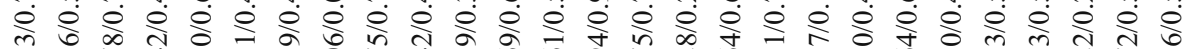

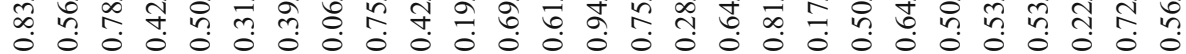

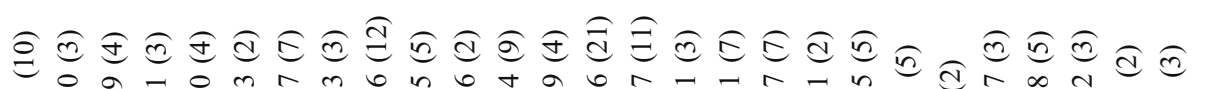

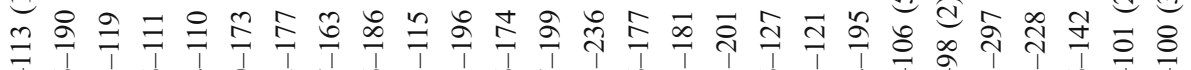

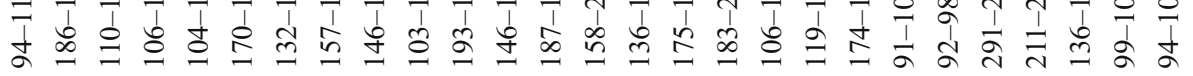

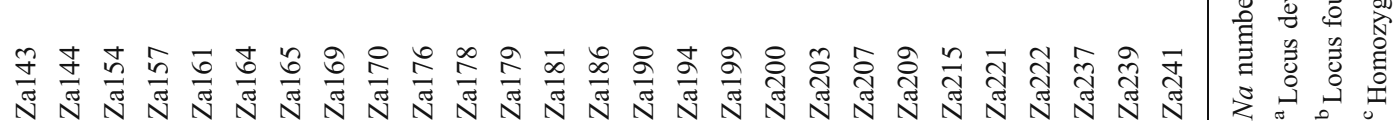


frequency based analyses but also in a coalescent theory framework for demographic inference.

Furthermore, to our knowledge, only a limited number of microsatellites ( $<15$ markers) were developed for $S$. lucioperca (Kohlman and Kersten 2008) and P. fluviatilis (Yang et al. 2009) to date. This set of microsatellites therefore provides an additional tool to researchers and practitioners in need of genetic markers for investigating the genetic structure and evolutionary history of wild populations and for fishery and aquaculture management. Finally, the success in cross-species amplification for the other percid species highlights the potential usefulness of the developed markers for a broader range of evolutionary, conservation and management studies in percids.

Acknowledgements We are very grateful to A. Crivelli for the $S$. lucioperca samples; to E. Corse, G. Olivari and M. Niveau for the $P$. fluviatilis samples; and to J. Bohlen for the Z. streber and G. schraetzer samples. We are also indebted to the ONEMA Alpes de Haute-Provence for their technical help and assistance in the field. Data used in this work were partly produced using the molecular genetic analysis technical facilities of the IFR119 'Montpellier Environnement Biodiversité'. This work was supported by Electricité de France.

Ethical standards The authors declare that the experiments described in this paper comply with the current French laws.

Conflict of interest The authors declare that they have no conflict of interest.

\section{References}

Altschul SF, Madden TL, Schäffer AA, Zhang J, Zhang Z, Miller W, Lipman DJ (1997) Gapped BLAST and PSI-BLAST: a new generation of protein database search programs. Nucleic Acids Res 25:3389-3402
Benjamini Y, Hochberg Y (1995) Controlling the false discovery rate - a practical and powerful approach to multiple testing. $\mathrm{J}$ R Stat Soc B 57:289-300

Borer SO, Miller LM, Kapuscinski AR (1999) Microsatellites in walleye Stizostedion vitreum. Mol Ecol 8:335-338

Danancher D, Izquierdo JI, García-Vásquez E (2008) Microsatellite analysis of relatedness structure in young of the year of the endangered Zingel asper (Percidae) and implications for conservation. Freshw Biol 53:546-557

Dubut V, Martin JF, Gilles A, van Houdt J, Chappaz R, Costedoat C (2009) Isolation and characterization of polymorphic microsatellite loci for the dace complex: Leuciscus leuciscus (Teleostei: Cyprinidae). Mol Ecol Resour 9:1179-1183

Kijas JM, Fowler JC, Garbett CA, Thomas MR (1994) Enrichment of microsatellites from the citrus genome using biotinylated oligonucleotide sequences bound to streptavidin-coated magnetic particles. Biotechniques 16:656-660, 662

Kohlman K, Kersten P (2008) Isolation and characterization of nine microsatellite loci from the pike-perch, Sander lucioperca (Linnaeus, 1758). Mol Ecol Resour 8:1085-1087

Laroche J, Durand JD (2004) Genetic structure of fragmented populations of a threatened endemic percid of the Rhône River: Zingel asper. Heredity 92:329-334

Mari S, Labonne J, Gaudin P (2002) A conservation strategy for Zingel asper, a threatened endemic percid of the Rhône basin. In: Collares-Pereira MJ, Cowx IG, Coelho MM (eds) Conservation of freshwater fishes: options for the future. Fishing News Books, Oxford, pp 149-156

Meglécz E, Costedoat C, Dubut V, Gilles A, Malausa T, Pech N, Martin JF (2010) QDD: a user-friendly program to select microsatellite markers and design primers from large sequencing projects. Bioinformatics 26:403-404

Rousset F (2008) GENEPOP'007: a complete reimplementation of the Genepop software for Windows and Linux. Mol Ecol Resour 8:103-106

Wirth T, Saint-Laurent R, Bernatchez L (1999) Isolation and characterization of microsatellite loci in the walleye (Stizostedion vitreum), and cross-species amplification within the family Percidae. Mol Ecol 8:1960-1962

Yang X, Wang C, Wang J, Ma Y, Yin J, Wu H (2009) Isolation and characterization of 12 polymorphic microsatellite loci in Eurasian perch (Perca fluviatilus L.). Conserv Genet Resour $1: 229-231$ 